\title{
Studies of Electrically-Exploded Conductors for Electric Armour Applications
}

\author{
P. Appelgren*, A. Larsson, P. Lundberg, M. Skoglund and L. Westerling \\ Swedish Defence Research Agency, FOI, Grindsjön Research Centre, SE-147 25 Tumba, Sweden \\ This paper presents experimental studies of current interaction with static copper rods. The results of the \\ static experiments are used to discuss the effects responsible for the disruption of shaped-charge jets in electric \\ armour. These effects include ohmic heating of, and current diffusion into, the rod as well as radial magnetic forces.
}

PACS numbers: 52.80.Qj

\section{Introduction}

When a powerful current is forced through a thin conductor, the conductor will be heated, melted and eventually evaporated. If the deposition of electric energy is sufficiently fast, the conductor will explode electrically. After the current interaction, the conductor may particulate and the fragments may transform into rings or thin discs. This effect is essential in an electric armour, where the current pulse is passed through a shaped-charge jet to disrupt it and prevent it from penetrating the base armour of the vehicle $[1,2]$. The radial dispersion of the jet significantly reduces its penetration performance into a target since the jet density decreases as well as the effective length of the jet fragments.

To study some basic phenomena of the disruption of a shaped charge jet, experimental studies of current interaction with static copper rods have been performed. Smooth as well as pre-notched copper rods were exposed for a high current pulse in a static setup. The notching was used to resemble the necking phenomena of a jet. Flash X-rays were used to depict the rod behaviour during and after the discharge. The voltage across and the currents through it were also monitored.

\section{Copper rod experiments}

A $1.4 \mathrm{mF}, 10 \mathrm{kV}$ pulsed power supply (PPS) was used in the copper rod experiments. The system inductance of $500 \mathrm{nH}$ is formed mainly by the closing switch, the electrodes and the rod. The system impedance $Z=\sqrt{L / C}$ where $L$ is the system inductance and $C$ is the capacitance, is $20 \mathrm{~m} \Omega$. The electrodes consist of $5 \mathrm{~mm}$ thick aluminium plates between which the copper rods are clamped. The electrodes are separated $100 \mathrm{~mm}$ allowing an experimental rod length of $90 \mathrm{~mm}$. The PPS voltage,

\footnotetext{
* corresponding author; e-mail: patrik.appelgren@foi.se
}

the voltage across the rod and the current through it, were monitored. Two flash X-ray tubes were used to depict the rod. Copper rods of $2 \mathrm{~mm}$ diameter were used. Some rods had five machined notches where the cross-section area is $75 \%$ of the original area i.e., the diameter at the notches is $1.73 \mathrm{~mm}$, see Fig. 1. Four experiments with notched rods were performed and one experiment with a smooth rod was performed as reference, Table. The PPS was charged to about $4.6 \mathrm{kV}$, i.e. $14.6 \mathrm{~kJ}$. The mass of the rod was $2.5 \mathrm{~g}$ for the experimental length of $90 \mathrm{~mm}$, i.e. about $6 \mathrm{~kJ} / \mathrm{g}$ was available for the rod disruption.

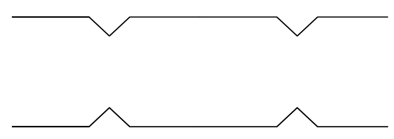

Fig. 1. Rod with machined notches.

\section{Results and discussion}

Due to the highly inductive circuit, the current pulse shape was roughly the same in amplitude and shape regardless whether a smooth rod or a rod with notches was tested. Figure 2a shows the current pulse from an experiment with a rod with notches. The vertical dashed lines mark the times of X-ray flashes.

The current action integral defined as

$$
J=\int_{t_{0}}^{t_{2}}\left(\frac{i(t)}{\pi r^{2}}\right)^{2} \mathrm{~d} t,
$$

where $i(t)$ is the current and $r$ is the radius, is calculated for the notch and for the full diameter, and is included in Fig. 2a. Melting of a copper rod initially at ambient temperature occurs for a current action integral value in the range of 8 to $9 \times 10^{16} \mathrm{~A}^{2} \mathrm{~s} \mathrm{~m}^{-4}$ and vaporization at a value of about $2 \times 10^{17} \mathrm{~A}^{2} \mathrm{~s} \mathrm{~m}^{-4}[3,4]$. Figure $2 \mathrm{~b}$ 

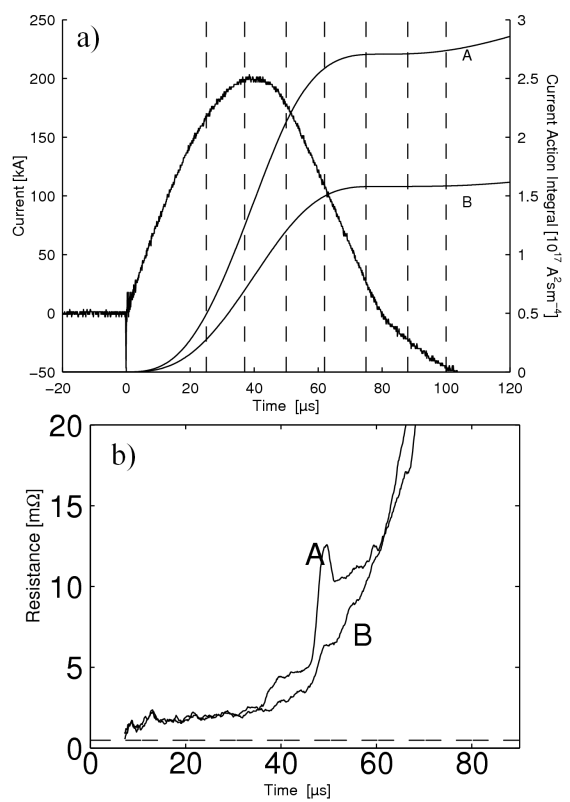

Fig. 2. (a) Current pulse and current action integral for (A) the notch and (B) the full diameter in static experiments. (b) The resistance of (A) the rod with notches and $(\mathrm{B})$ the smooth rod.

TABLE

Experimental settings

\begin{tabular}{c|c|c}
\hline \hline $\begin{array}{c}\text { Energy in } \\
\text { capacitors }[\mathrm{kJ}]\end{array}$ & $\begin{array}{c}\text { Rod } \\
\text { geometry }\end{array}$ & $\begin{array}{c}\text { X-ray } \\
\text { times }[\mu \mathrm{s}]\end{array}$ \\
\hline 15.0 & notch & $50 / 200$ \\
14.4 & notch & $37 / 75$ \\
14.7 & notch & $25 / 62$ \\
14.6 & notch & $88 / 100$ \\
14.6 & smooth & $75 / 100$
\end{tabular}

shows the measured resistance of the rods where the trace marked A is the rod with a notch and B is for a smooth rod. The resistances are identical up to about $35 \mu \mathrm{s}$ when the resistance of the rod with notches suddenly increases i.e., it melts. At about $47 \mu$ s there is another step associated with the notch explosion. At the same time the smooth rod has a step in resistance associated with melting. This is consistent with the values of the action integrals for melting and explosion. A montage of a series of flash X-ray pictures taken at times according to Table, is shown in Fig. 3a. At time $37 \mu$ s material around the upper notch has separated indicating melting. At times from $50 \mu$ s material is ejected from the notches indicating explosion. The part of the rod without notches begins to deform at $50 \mu \mathrm{s}$. Figure $3 \mathrm{~b}$ shows the smooth and a rod with notches at times 75 and $100 \mu \mathrm{s}$. At the times 75 and $100 \mu \mathrm{s}$ both the rod with notches and the smooth one are disrupted. At $75 \mu \mathrm{s}, 7.5 \mathrm{~kJ}$ (smooth rod) and $8.2 \mathrm{~kJ}$ (rod with notches) has been deposited in the rod.
The radial velocity of the material from the exploding notches can be estimated from the X-ray pictures. The radial velocity is calculated for the explosion of the top notch at time $62.5 \mu \mathrm{s}$ in Fig. 3b. The diameter has increased from $2 \mathrm{~mm}$ at time 37.5 to $14 \mathrm{~mm}$ at time $62.5 \mu \mathrm{s}$ corresponding to a mean velocity of $240 \mathrm{~m} / \mathrm{s}$. A corresponding measurement of the smooth lower part of the rod yields a velocity of $25 \mathrm{~m} / \mathrm{s}$, i.e. significantly lower than that of the notched part.

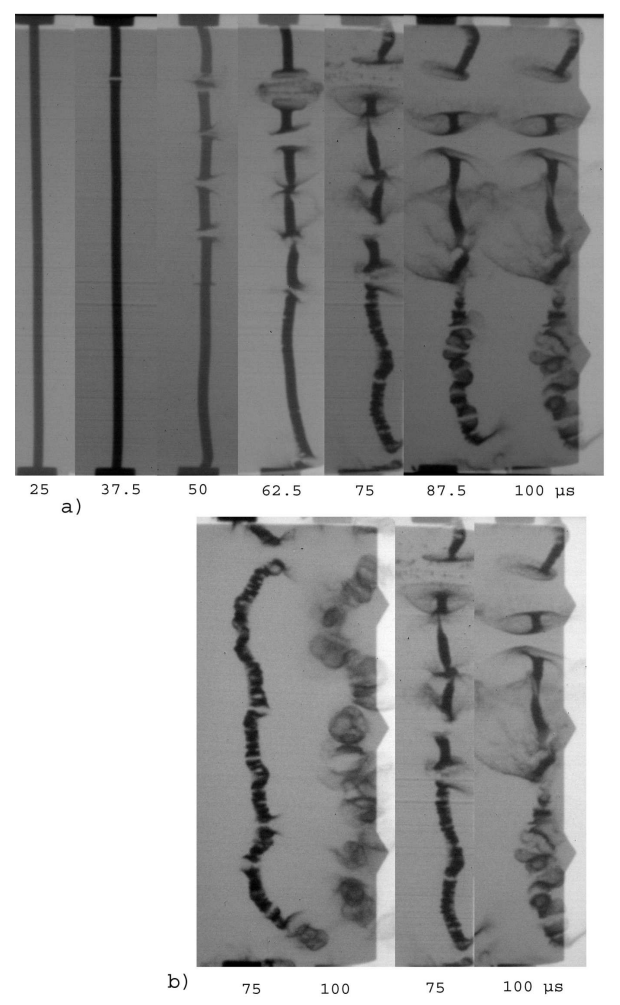

Fig. 3. (a) Montage of X-ray pictures of a copper rod with notches subject to a current pulse. (b) X-ray pictures of a smooth (left) copper rod and a rod with notches (right) at times 75 and $100 \mu \mathrm{s}$.

\section{Conclusions}

In electric armour a radial expansion of the jet is necessary to reduce its penetration in the base armour. A practical distance between the electrodes and the base armour of a vehicle would be 0.2 to $0.4 \mathrm{~m}$. A jet fragment with axial velocity of $6 \times 10^{3} \mathrm{~m} / \mathrm{s}$ travels $300 \mathrm{~mm}$ in $50 \mu$ s and assuming a radial velocity of $240 \mathrm{~m} / \mathrm{s}$, its radius would have increased by $12 \mathrm{~mm}$ when hitting the base armour. A slower fragment of $3 \times 10^{3} \mathrm{~m} / \mathrm{s}$ would have increased to twice that value. In these experiments about $8 \mathrm{~kJ}$ was deposited in the rod and since a jet of a medium shaped charge has ten times the mass of the rod, one may assume that $80 \mathrm{~kJ}$ is required to the disrupt the jet. Another important requirement is that the energy is deposited sufficiently fast. To explode a jet fragment 
it is necessary to reach sufficiently high current action integral value within the time the current passes through the fragment. However, a jet is not at ambient temperature but has a temperature of about $800 \mathrm{~K}$ [5], which significantly reduces the current action integral required for explosion. Other effects than ohmic heating are also present when a jet passes an electric armour, e.g. magnetic forces acting on the jet in between the electrodes and at exit of the electrode region. In the X-ray pictures, it is seen that the Lorentz force clearly pushes the rod to the right. The impulse received by a fragment could possibly give a shape charge jet a velocity sideways that will prevent it from hitting the same spot as previous jet fragment and hence smearing out the jet over a larger target surface.

\section{Acknowledgments}

The work was supported by the Swedish Armed Forces.

\section{References}

[1] E.H. Walker, U.S. Army Ballistic Research Laboratory, BRL-MR-2309 (1973).

[2] G.A. Shvetsov, A.D. Matrosov, Proc. Megagauss VII Conference, Sarov, Russia, August 5-10, 1996, RFNC-VNIIEF (1997).

[3] T.J. Tucker, R.P. Toth, Sandia Laboratories, SF 1004DF(2-74). SAND-75-0041 (1975).

[4] H. Knoepfel, Pulsed High Magnetic Fields, North-Holland, Amsterdam 1970.

[5] W.P. Walters, J.A. Zukas, Fundamentals of Shaped Charges, Wiley 1989. 